\title{
THE MANIFOLD OF ELLIPTICAL GALAXIES
}

\author{
S. Djorgovski \\ Harvard-Smithsonian Center for Astrophysics \\ 60 Garden St. \\ Cambridge, MA 02138, USA.
}

\begin{abstract}
Global properties of elliptical galaxies, such as the luminosity, radius, projected velocity dispersion, projected luminosity density, etc., form a twodimensional family. This "fundamental plane" of elliptical galaxies can be defined by the velocity dispersion and mean surface brightness, and its thickness is presently given by the measurement error-bars only. This is indicative of a strong regularity in the process of galaxy formation. However, all morphological parameters which describe the shape of the distribution of light, and reflect dynamical anisotropies of stars, are completely independent from each other, and independent of the fundamental plane. The $M / L$ ratios show only a small intrinsic scatter in a luminosity range spanning some four orders of magnitude; this suggests a constant fraction of the dark matter contribution in elliptical galaxies.
\end{abstract}

The problem of the "minimal manifold of galaxies" is, how many and which physical quantities are necessary and sufficient to describe a family of normal galaxies? Such description of important physical variables and relations between them is important for our understanding of the structure, formation, and evolution of galaxies. Brosche (1973) was perhaps the first to clearly state the problem in this way. Many, but not all, properties of elliptical galaxies correlate with luminosity (cf. the review by Kormendy 1982, and references therein), and in almost all cases there is a residual scatter, not accountable by the measurement errors, and indicative of a presence of "hidden parameters". The previous studies by Tonry \& Davis (1981), Terlevich et al. (1981), Efstathiou \& Fall (1984), Lauer (1985), and Djorgovski, Davis \& Kent (1985) indicated that there is more than one important quantity (the one assumed to be the luminosity), but there was no clear understanding or agreement as what the second parameter may be, or whether there are more than two. In more recent attacks on this problem, Burstein et al. (1986) and Dressler et al. (1986), and Djorgovski \& Davis (1986ab) used new, large, homogeneous data sets, and independently reached essentially the same solutions. I will briefly describe here the results, presented in more detail by Djorgovski \& Davis (1986b).

The existence of large and homogeneous data base was necessary for the solution of the minimal manifold problem. We used morphological parameters, radii and magnitudes from the CCD surface photometry survey of $\sim 260$ early-type 
galaxies by Djorgovski (1985a), and line strength and central velocity dispersion measurements by Tonry \& Davis (1981) and the compilation by Whitmore et al. (1985). The details of surface photometry survey, data reductions, error estimates and calibrations are given by Djorgovski (1985a; and in preparation). The selection effects are well understood. The sample excludes diffuse dwarfs, which are probably a completely different family of galaxies (cf. Wirth \& Gallagher 1984, Kormendy 1985, or Sandage et al. 1985).

In order to parametrize the morphology of galaxies in the sample, a consistent radial scale is needed. This is a non-trivial problem, and we defined and used several different scales, all of them derived from our surface photometry profiles, but independent of any particular isophotal threshold, and thus free of magnitude calibration errors. The results were essentially the same with all radial scales which we used, but slightly better fits are obtained with larger radii: the relations described below are robust, but they are indicative of more global, rather than central, properties of galaxies. In this report, I will use the values of $r_{e}$ (actually, a semimajor axis, not radius), obtained from the fits of surface brightness to de Vaucouleurs' $r^{1 / 4}$ formula. This is operationally a parameter from the fit, rather than a half-light radius. All quantities used here were measured at $r_{e}$, or within the elliptical isophote whose semi-major axis is $r_{e}$. For each galaxy, we thus have measurements of radius, magnitude, mean and local surface brightness, slope of the surface brightness profile, ellipticity, ellipticity gradient, and isophotal twist rate. The magnitudes are defined in appropriate elliptical-isophote apertures; both magnitudes and surface brightness are in the (red) $r_{G}$ band, defined by Djorgovski (1985b). In addition, there are central velocity dispersion $(\sigma)$ and line strength measurements from the literature, though not for all galaxies in the survey. There are carefully estimated error-bars for all quantities.

We employed a multi-bivariate statistical analysis in our study, and we intend to repeat the analysis by using different multivariate methods (PCA, MDRACE). We started by investigating the known distance-indicator relations (luminosity or radius $v s$. velocity dispersion, $\sigma$, or mean surface brightness), and correlating their residuals with other quantities. It was immediately apparent that the residuals of $\mathrm{L}-\sigma$ and $\mathrm{R}-\sigma$ relations correlate well with the mean surface brightness $(\langle\mu\rangle$, in the usual logarithmic, magnitudes-per-square-arcsec form), and vice versa. On the other hand, $\sigma$ and $\langle\mu\rangle$ do not correlate at all! This, indeed, was the solution: linear combinations of $\log \sigma$ and $\langle\mu\rangle$ with $\operatorname{logs}$ of $L$ or $R$ produce excellent fits, with no residual scatter, i.e., not accountable by the measurement errors. A possibility of surface brightness as a "second parameter" in the Faber-Jackson relation was already indicated by de Vaucouleurs \& Olson (1982), and Lauer (1985) emphasized possible significance of the three-dimensional luminosity density as a parameter in the core properties of ellipticals. The new distance-indicator relations, which at the same time are the equations of a plane in the $\mathrm{L}$ (or R) $-\sigma-\langle\mu\rangle$ parameter space, are:

$$
\begin{gathered}
M\left(r_{e}\right)=-8.62(\log \sigma+0.10\langle\mu\rangle)+16.14 \\
\text { or : } \quad L \sim \sigma^{3.45}\langle S B\rangle^{-0.86} \\
\log r_{e}=1.39(\log \sigma+0.26\langle\mu\rangle)-6.71 \\
\quad \text { or : } \quad R \sim \sigma^{1.39}\langle S B\rangle^{-0.90}
\end{gathered}
$$


Here $\langle S B\rangle$ denotes the mean surface brightness in linear flux units, and $r_{e}$ is measured in pc ( $h=1$ was assumed). The magnitudes are in the red $\left(r_{G}\right)$ bandpass. The power-law coefficients of $\sigma$ and $\langle\mu\rangle$ are uncertain by about $10 \%$. These distance-indicator relations represent the fundamental plane of elliptical galaxies viewed edge-on. The Eqs. (1) and (2) were derived independently, and thus do not transform into each other exactly. The Eqs. (2ab) are the better ones, since the magnitudes are afflicted by our zero-point calibration errors, and the radii are not. A good graphical representation in terms of observable quantities is the plane defined by $\sigma$ and $\langle\mu\rangle$, as shown in Figure 1.

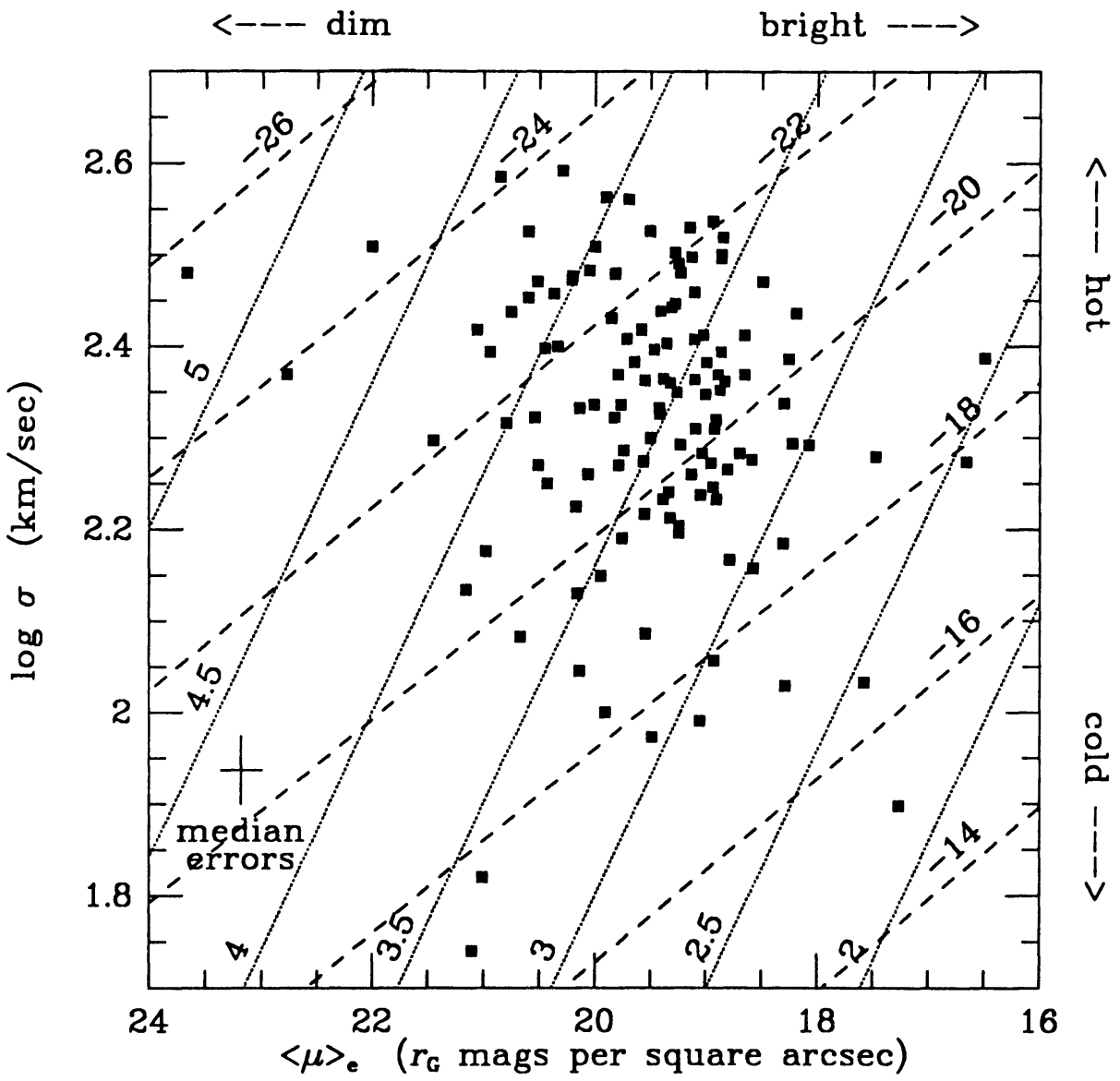

Figure 1: The fundamental plane of elliptical galaxies, shown through the observable quantities, projected central velocity dispersion, $\sigma$, measured in $\mathrm{km} \mathrm{s}^{-1}$, and mean surface brightness within the $r_{e}$ isophote, $\langle\mu\rangle$, measured in $r_{G}$ magnitudes per square arcsec. The dashed lines indicate loci of equal absolute magnitudes (within the $r_{e}$ isophote, in the $r_{G}$ band), and the dotted lines indicate loci of equal semimajor axis $r_{e}$, measured in $h^{-1}$ pc, and expressed as logarithms (base 10). A Virgocentric infall model with $V_{I N F}=400 \mathrm{~km} \mathrm{~s}^{-1}$ was assumed in computing the absolute luminosities and radii. The error-bar in the lower left indicates the median errors for the points. 
Introduction of line strength as a third parameter does not improve the fits significantly. Line strengths and colors are very well correlated with $\sigma$, and can be viewed as additional axes of the parameter space in which this plane is defined. The residuals of new relations do not correlate significantly with any other morphological or spectroscopic quantity, indicating that two dimensions provide an adequate and exhaustive description of the global properties of ellipticals. The plane thickness is completely contained in the present measurement errors, and any cosmic broadening must be very small, on the level of a few percent, or less. The measurement of the intrinsic thickness is is an important target for the future investigations, as it would probe directly the "noise of galaxy formation".

An important question is, how linear are the Eqs. $(1-2)$, or, how flat is the "fundamental plane"? First, there is no a priori reason why the equations should be pure power-laws, even though the plane appears to be flat within the present measurement errors. A possible physical mechanism which may introduce curvature at the "bright" end, or individual deviations from the plane, could be dissipationless mergers. This can be tested by looking at the positions of apparent cannibals (e.g., galaxies with shells) with respect to the fundamental plane. The very existence of such global regularity, and over several orders of magnitude in luminosity or radius, suggests that elliptical galaxies are formed by a single process, probably dissipative and primordial, but the formation by a large number of mergers is still a possible alternative. Perhaps N-body simulations can be used to test this idea, whether and in which conditions the final locus of multiple mergers is a plane in the L (or R) $-\sigma-\mu$ space, and if so, whether the plane tilt is as observed. It is still possible that there are small differences in the tilts and/or intercepts of the fundamental plane in different large-scale environments, which would reflect, perhaps, some real fluctuations in the process of galaxy formation. Any such deviations, or curvature, would have a considerable importance for the use of Eqs. $(1-2)$ as distance indicators: systematic deviations in clusters at different distances (when generally different portions of the luminosity function are sampled) would show up as large peculiar velocities.

It is possible that galaxies in all cluster/field environments lie on the same plane, but populate different portions of it. This may be investigated by looking at the distributions perpendicular to the luminosity axis in the plane, thus factoring out any possible differential selection effects. Galaxian activity (radio power, presence of LINER nuclei, etc.) may also be correlated with the positions in the plane: such correlations, if they exist, could be smeared by projecting them at any of the observable axes, and thus could have passed undetected so far.

Projected velocity dispersions should be influenced by any dynamical anisotropies that may be present. Such anisotropies give rise to the flattening and/or triaxiality of E-galaxies, and thus are reflected in their ellipticities $(\epsilon)$, ellipticity gradients, and isophotal twists. Radial-to-tangential anisotropy modifies the radial slope of a surface brightness profile. However, none of these shape parameters correlate with velocity dispersion, or any other global property! Moreover, the shape parameters do not correlate between themselves, either. There are also no correlations between any of the structural properties and kinematic variables (maximum rotational velocity, $V_{\max }$, its ratio with the mean velocity dispersion, $\left.V_{\max } /\langle\sigma\rangle\right)$ from a subset of galaxies of Davies et al. (1983), except for the known very weak correlation between $V_{\max }$ and ellipticity. In particular, ellipticity does 
not correlate with either $\sigma$ or $\langle\mu\rangle$. This renders very difficult or impossible the tests for intrinsic shapes of ellipticals, proposed by several authors in the past: $\sigma$ and $\langle\mu\rangle$ were expected to correlate with $\epsilon$ in the oblate case, and anticorrelate in the prolate case, but a pure scatter is all that is observed.

Since these dynamical quantities are not related to the properties determined by the fundamental plane, which presumably reflect the process of galaxy formation, they must be determined by some other process, or reflect the initial conditions present in the protogalactic (protocluster?) material. There are some indications that the ellipticities and/or galaxy orientations may be related to the properties of the large-scale structure in which they live (Strom \& Strom 1978, Djorgovski 1983, and references therein). If I am permitted a crude speculation, and the indications mentioned above are true, then dynamical anisotropies, now frozen in the kinematics of stars, reflect the dynamical anisotropies of the protogalactic material (set, perhaps, by the process of protocluster formation?), whereas the the process of actual galaxy formation, reflected, presumably, in the fundamental plane, determines how is that material going to be cut and assembled in individual galaxies, without any regard for its internal velocity anisotropy. Alternatively, the kinematic anisotropies may be acquired or modified in a stochastic way after the galaxies form, by tidal torquing and/or mergers.

Thus, the elliptical galaxies are a " $2+n$ " parameter family, defined by the following observable (projected) quantities: (1) velocity dispersion, (2) surface brightness, and (3,etc.) a variety of shape/anisotropy parameters. A possible interpretation in physical terms is that the two principal parameters are the depth of the potential well (1), and the mean density (2), whereas the multitude of subtle dynamical anisotropies $(3, \ldots)$ determine the details of internal dynamics, and thus the shapes. Note, however, that a full dynamical interpretation of all these observational quantities is afflicted by the projection effects.

In most studies to date, mass-to-light $(M / L)$ ratios have shown a considerable scatter, some of which was (correctly) attributed to the poor and heterogeneous state of photometry available at the time. I have computed the $M / L$ ratios measured within the $r_{e}$ isophote by using the Poveda's formula (cf. Tonry \& Davis 1981); this formula is but a gross approximation: it assumes a spherical galaxy, in which both light and mass follow exactly the $r^{1 / 4}$ law, and with an isotropic velocity dispersion tensor; all of these assumptions are wrong in differing degrees for all real galaxies, and we do not have the mean, but rather projected central velocity dispersions. Thus, a straight application of this formula will inevitably introduce some scatter in the computed $M / L$ ratios.

Figure 2 shows the histogram of $M / L$ ratios. Note the relatively small spread: the r.m.s. of the histogram is only 0.21 (in log); the median error of measurement is 0.17 . This indicates an intrinsic broadening of only about $30 \%$, which should also incorporate any differential shape and anisotropy effects, ignored in my simple computation. Figure 3 plots the computed $M / L$ ratios $v s$. the luminosity. The $M / L$ changes by certainly much less than a factor of ten over some four orders of magnitude in luminosity, and may even be constant. In fact, the only correlation involving $M / L$ which is present in our data is with the velocity dispersion, which is well-known (Faber \& Jackson 1976, and references therein). The relative constancy or universality of $M / L$ may imply that the baryons and the dark matter are well 
mixed on the scales of galaxies, or larger - otherwise, galaxies in different places could have substantially different relative fractions of the dark material.

To summarize, we see a strong regularity in a contrast with a strong cosmic scatter: On one side, there is a set of global properties (luminosity, mass, radius, density, velocity dispersion, metallicity, separately or in various mutual combinations), well described by the thin fundamental plane. On the other hand, there is a set of shape parameters (ellipticities, ellipticity gradients, isophotal twists, surface brightness slopes, rotational properties), reflecting internal dynamical structures of ellipticals, all of which are independent from the first family, and generally independent between themselves as well. The manifold of observable properties of elliptical galaxies is thus split into two distinct sets, one two-dimensional and highly orderded, and the other multi-dimensional, and quite chaotic. The (global) $M / L$ ratios vary very little, and may even be constant. We need a theory of galaxy formation which can account for all this.

Historically, accounting for the shape parameters, all of which were clearly statistically independent, caused some confusion, and prevented some authors (myself included) from seeing forest for the trees: in multivariate analyses there were far too many significant eigen-values and eigen-vectors... Another mistake was to assume that the luminosity is the first parameter: we now see that it is a product of $\sigma$ and $\mu$. One may define the luminosity as one of the fundamental plane axes, but then the "second" parameter, perpendicular to that axis, is not anything directly observable or interpretable. Finally, there was always the ideology of the second parameter, implicitly assuming or suggesting that there are only two, and some authors even went out to state without a really good substantiation that the ellipticals are a two-parameter family, since that was thought to be the desirable answer, but which we now know is wrong, or rather, an incomplete answer.

The question which remains is, how do galaxies with other morphologies behave? A preliminary investigation with a sample of $\sim 50$ S0 galaxies, but only for 18 of which we have velocity dispersions, shows that their global properties also form a two-dimensional family, and that the equations (tilt) of their fundamental plane are very similar, and possibly identical, to the plane of ellipticals. Their shape parameters do not correlate with the global properties either. Thus, there seems to be a fundamental continuity between ellipticals and S0's. Whitmore (1984) reached a conclusion that the spirals also form a two-dimensional family, but the observables are quite different there (see also Watanabe, Kodaira \& Okamura 1985). There may be some indications of the presence of "second" parameter(s) in the Tully-Fisher relation, perhaps in analogy with our Eqs. (1ab). We may be probing the same fundamental relation for all galaxies, but the surface quirks or morphology determine our observables, and make direct comparisons difficult.

Finally, a note on the dynamical models of ellipticals. The distribution of light in elliptical galaxies shows a wide variety of shapes, both in azimuthal and radial sense. The radial surface brightness profiles show a considerable and significant variety of shapes - the ellipticals are not well described by the $r^{1 / 4}$ law, Hubble law, or any other simple formula, or by the King, Binney, or Jaffe models. There is also a variety of ellipticity gradients and isophotal twists. This means that any realistic dynamical model of elliptical galaxies must incorporate several structure parameters, and reproduce this variety; they have to be very complex. But 
the fate of a dynamicist is even worse, if the ellipticals have substantial amounts of dark material, whose radial and azimuthal distribution is completely unknown at the present: any self-consistent dynamical models, in which stars provide both light and mass, are then simply inadequate. All this requires good-quality data, and it may be possible to provide some additional constraints on the true isopotential surfaces from detailed observations of X-ray coronae.

I would like to thank to the staff of Lick Observatory for their help during the surface photometry survey, on which this work is based, and to Marc Davis, who paid the bills. I acknowledge useful conversations with Marc Davis, Ivan King, Sandra Faber, Roger Davies, and many others. This work was supported in part by the NSF grant AST84-19910 to M. Davis, and a Harvard Junior Fellowship to the author.

\section{REFERENCES:}

Brosche, P. 1973, Astron. Astrophys. 23, 259.

Burstein, D., Davies, R., Dressler, A., Faber, S., Lynden-Bell, D., Terlevich, R., and Wagner, M. 1986, in Distances to Galaxies and Deviations from the Universal Expansion, B. Madore and B. Tully (eds.), p. 123. Dordrecht: D. Reidel.

Davies, R., Efstathiou, G., Fall, M., Illingworth, G., and Schechter, P. 1983, Astrophys. J. 266, 41.

de Vaucouleurs, G., and Olson, D. 1982, Astrophys. J. 256, 346.

Djorgovski, S. 1983, Astrophys. J. Lett. 274, L7.

Djorgovski, S. 1985a, Ph.D. Thesis, University of California, Berkeley.

Djorgovski, S. 1985b, Publ. Astr. Soc. Pacific 97, 1119.

Djorgovski, S., Davis, M., and Kent, S. 1985, in New Aspects of Galaxy Photometry J.-L. Nieto (ed.), Lectures in Physics 232, p. 257, Springer Verlag.

Djorgovski, S., and Davis, M. 1986a, in Distances to Galaxies and Deviations from the Universal Expansion, B. Madore and B. Tully (eds.), p. 135. Dordrecht: D. Reidel.

Djorgovski, S., and Davis, M. 1986b, Astrophys. J., in press.

Dressler, A., Lynden-Bell, D., Burstein, D., Davies, R., Faber, S., Wagner, M., and Terlevich, R. 1986, Astrophys. J., in press.

Efstathiou, G., and Fall, M. 1984, M.N.R.A.S. 206, 453.

Faber, S., and Jackson, R. 1976, Astrophys. J. 204, 668.

Kormendy, J. 1982, in Morphology and Dynamics of Galaxies, proceedings of the 12th Saas-Fee Advanced Course, Geneva Observatory publication.

Kormendy, J. 1985, Astrophys. J. $295,73$.

Lauer, T. 1985, Astrophys. J. 292, 104.

Sandage, A., Binggeli, B., and Tamman, G. 1985, Astron. J. 90, 1759.

Strom, S., and Strom, K. 1978, Astron. J. 83, 732.

Terlevich, R., Davies, R., Faber, S., and Burstein, D. 1981, M.N.R.A.S. 196, 381.

Tonry, J., and Davis, M. 1981, Astrophys. J. 246, 666.

Watanabe, M., Kodaira, K., and Okamura, S. 1985, Astrophys. J. 292, 72.

Whitmore, B. 1984, Astrophys. J. 278, 61.

Whitmore, B., McElroy, D., and Tonry, J. 1985, Astrophys. J. Suppl. Ser. 59, 1.

Wirth, A., and Gallagher, J. 1984, Astrophys. J. 282, 85. 


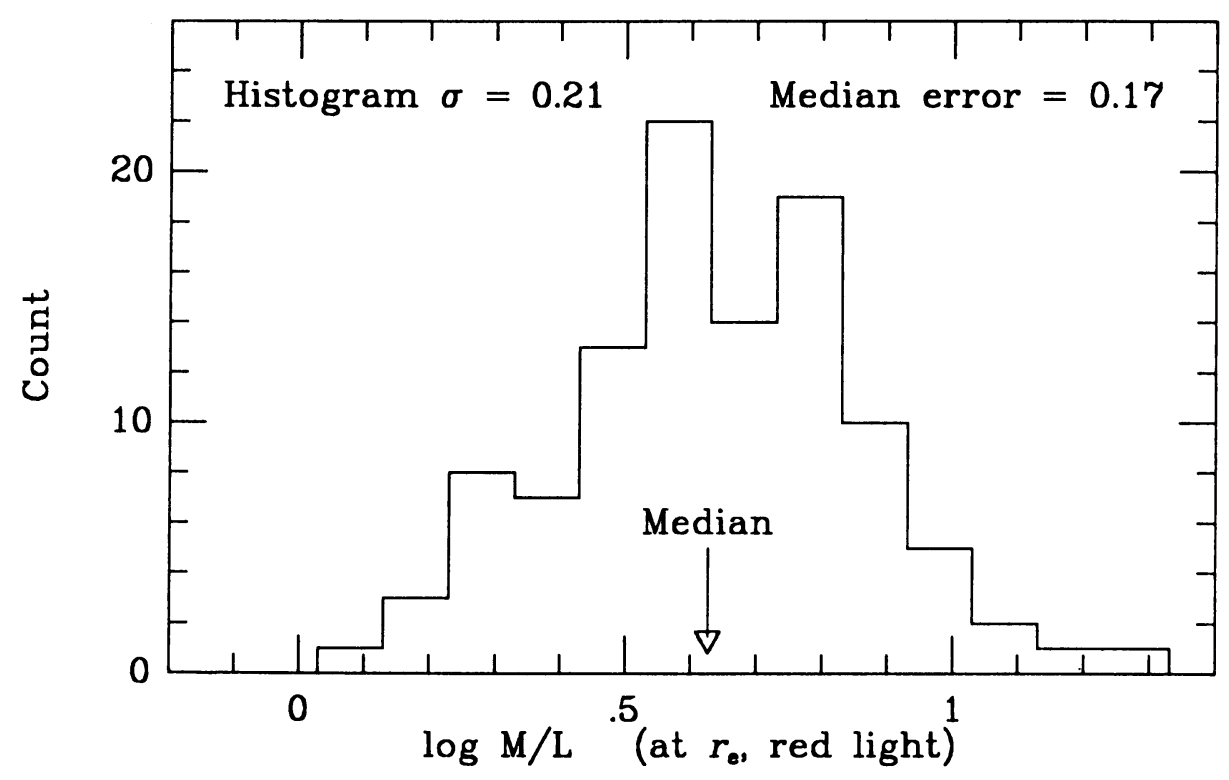

Figure 2: The distribution of (red) $M / L$ ratios, computed with the Poveda's formula, within the $r_{e}$ isophote. Most of the spread is due to the measurement errors, and the residual scatter is estimated to be $\sim 30 \%$, which must also include any possible scatter caused by the application of an approximate formula.

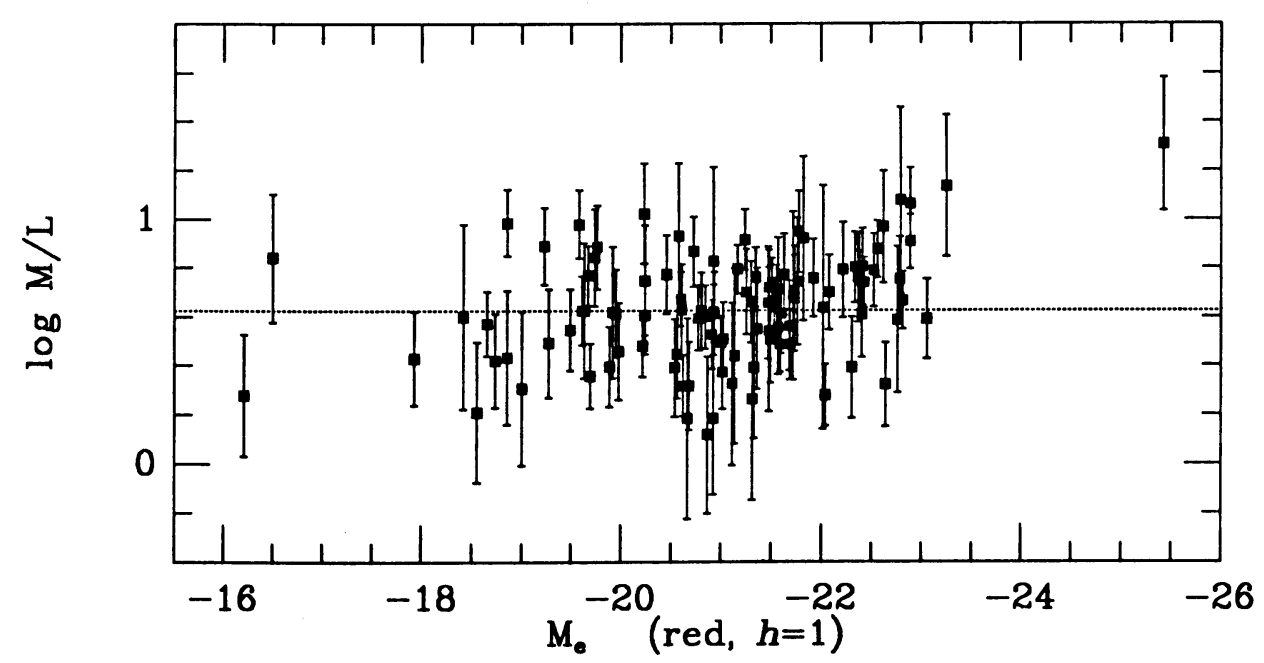

Figure 3: The $M / L$ ratios plotted against the absolute magnitude $\left(\mathrm{M}_{e}\right)$, in the $r_{G}$ band, within the $r_{e}$ isophote. A Virgocentric infall model with $400 \mathrm{~km} \mathrm{~s}^{-1}$, and $h=1$ were used for computation. Dotted line indicates the median $M / L$. There is no indication that $M / L$ varies with luminosity. 


\section{DISCUSSION}

Schechter: My understanding of your "fundamental plane" is that you can use any two parameters to predict a third. I would be interested in seeing luminosities and surface brightnesses used to predict velocity dispersions, and then observed minus predicted dispersions plotted versus apparent ellipticity. This might produce a better correlation with ellipticity than either dispersion or surface brightness alone. Have you made such a plot?

Djorgovski: Not directly, but this may be equivalent to introducing the ellipticity in my residual fits. This did not help much. It is a good idea, and I will try it, but I doubt that it will show anything, since there is absolutely no correlation between the ellipticity and surface brightness, velocity dispersion, or any linear combination of the two.

de Zeeuw: There are a few posters outside (Statler, Levison) that show dynamical models with a variety in their velocity dispersion tensors that is possibly larger than you see in real ellipticals.

Djorgovski: Well, good, but the observed variety is such that constraining any models may be very difficult.

White: I would take issue with your statement that the fundamental plane of $L$ or $r$ as a function of $\langle\mu\rangle$ and $\sigma$ is only a few percent thick. From your data the only safe conclusion is that the plane is thinner than the observed scatter. What is the rms observed scatter of $\log L$ and $\log r$ about the predictions of your relations?

Djorgovski: I cannot really answer that offhand, since there are projection effects, and error correlations. When the new relations are used as distance indicators, the errors of distances expressed in magnitudes are about $0 .^{m} 4$, or $\sim 20-25 \%$. However, please recall that the observed scatter is now given entirely by the error bars, that is, $\chi^{2} \sim 1$. This leaves only a little space for intrinsic broadening.

King: First, a comment: you should not say that $\sigma$ measures the depth of the potential well. It doesn't; in the King models (which, incidentally, don't fit your profiles either) the model parameter $W_{0}$ explicitly measures the amount by which $\sigma$ does not measure the depth of the potential well. Second, a question: why didn't you use principal-component analysis, instead of plotting residuals? And a final question, can't you get line strengths from the velocity-dispersion papers?

Djorgovski: About velocity dispersions-yes, you are quite right, but I hope that my intuitive meaning was clear. Then, I did use the principal component analysis some three years ago with the fine data by Steve Kent. I got some solutions, but they meant nothing to me, since there were too many significant eigen-values (this is still the case). The overabundance of significant dimensions in the total set of observable parameters prevented me from seeing the fundamental plane which unites a subset of fundamental properties, as expanded in my talk. In other words, I did not see the forest for the trees, and a similar thing happened to some other people who used the principal component analysis on this problem. Even if one does apply the principal component analysis correctly (and Efstathiou \& Fall addressed 
some of the important problems), the formal solutions may be quite non-intuitive. My intuition worked much better when I started from the other end, with multibivariate statistics. Finally, I did use the line strength measurements from Tonry \& Davis, but they are not very good by the present standards, and for a variety of reasons I believe that introduction of line strengths does not help much in this particular problem.

Richstone: If isophotal twists are observed equally often in low and high luminosity ellipticals, and if we believe (as Davies suggests we ought to), that low $L$ ellipticals are oblate isotropic rotators, then what's the logic of using isophotal twists to identify triaxial objects?

Djorgovski: The bulges and the low-luminosity ellipticals are similar in many ways, but not all: For example, the bulges often show boxy isophotes, but the lowluminosity ellipticals do not. Besides, the observed $(V / \sigma)^{*}$ vs. luminosity correlation is very noisy, and some bulges (and certainly ellipticals) may well be triaxial.

Whitmore: My first comment is a note of caution addressed toward Ivan King's suggestion that the line strength index determined from the Fourier quotient technique be used to estimate metallicity. This will not be useful since the line strength index is on a relative scale, determined by the template star used to make the reductions, rather than on an absolute scale. Since everyone uses different template stars, these are all on a different system.

My second comment is for George. In the interest of trying to compare your twodimensional parameter space for elliptical galaxies with the two-dimensional space I found for spiral galaxies, do you have any color information for your sample?

Djorgovski: I did not measure colors, and there is no sufficiently large, homogeneous data base in the literature. To the extent that colors measure metallicity in Egalaxies, my remarks to Ivan about line strengths still apply. Faber et al. will probably be able to do a better job than what I can.

Davies: The size of the isophote twist as a function of luminosity alone does not indicate the degree of triaxiality. Isophote twists can also be caused by tidal effects, so that if one believes that lower luminosity ellipticals are oblate, then statistically one would expect to find few isophote twists at small radii in them compared to their brighter brothers, i.e., one needs to attach a radius to the isophote twists to make this comparison.

Djorgovski: Quite right. As you know, I have used several different radial scales, and the results are the same at all fiducial radii. Unfortunately, I do not have a sample of bulges. 